\title{
A Realizability Interpretation for Classical Analysis
}

\author{
Henry Towsner
}

May 20, 2005

\begin{abstract}
We present a realizability interpretation for classical analysis-an association of a term to every proof so that the terms assigned to existential formulas represent witnesses to the truth of that formula. For classical proofs of $\Pi_{2}$ sentences $\forall x \exists y A(x, y)$, this provides a recursive type 1 function which computes the function given by $f(x)=y$ iff $y$ is the least number such that $A(x, y)$.
\end{abstract}

\section{Introduction}

Although both classical and intuitionistic arithmetic prove the same $\Pi_{2}$ sentences, proofs in the intuitionistic version generally provide more information. The CurryHoward isomorphism associates them with realizing $\lambda$ terms, which associate numerical witnesses to existential quantifiers and appropriate functionals to strings of quantifiers.

[Avigad, 2000] demonstrates a method of extending this realization to classical arithmetic to find numerical witnesses to $\Sigma_{1}$ sentences and type 1 functions witnessing $\Pi_{2}$ sentences. This method of witness extraction was derived from the composition of an embedding of classical logic in intuitionistic logic, the Friedman-Dragalin translation (first described in [Friedman, 1978] and [Dragalin, 1980]), and the Curry-Howard isomorphism.

In this paper we extend this method to second order classical arithmetic. As with Avigad's version, the actual embedding of classical logic in intuitionistic logic is unusually simple; in particular, unlike the double-negation translation, an atomic formula $\phi$ in classical logic is unchanged in the intuitionistic embedding. This leads to a different type of equivalence between the theories: if we can prove $\phi$ in classical logic then $\phi\urcorner\urcorner$ can be proven in intuitionistic logic. By contrast, under the embedding used here, we will be able to prove instead that $(\neg \phi)^{E} \vdash \perp$.

The embedding used here is simplified by not allowing implication or universal quantifiers in the classical language, instead building them in the usual way from negation, disjunction, and existential quantifiers. This means that, for example, $\forall x \phi$ is embedded is $\neg \exists x \neg \phi$ : no universal quantifiers appear in the range of the embedding. 
In order to find $\lambda$ terms corresponding to intuitionistic proofs, the $H R O^{2}-m r$ realizability given in [Troelstra, 1973] will be used, based on Kreisel's modified realizability presented in [Kreisel, 1959] and [Kreisel, 1962]. The system $H R O^{2}$ encodes functionals as numbers and the modified realizability associates a type to each formula of $H A^{2}$ and a particular term of that type to each proof of the formula.

We will show that each proof of a $\Sigma_{1}$ formula $\exists x A(x)$ in $H A^{2}$ can be converted into a term $t$ of $H R O^{2}$ such that analysis proves that $t$ is defined and satisfies $A$ for every value of any parameters appearing in $A$.

\section{Preliminaries}

A Tait style calculus based on the one in [Schwichtenberg, 1977] will be used for $P A^{2}$. The primary difference is that $\neg$ is taken as a connective, rather than a shorthand for the negation-normal form. Atomic formulae will be either of the form $s=t$ or $X t_{1} \ldots t_{n}$ (where $s, t, t_{1}, \ldots, t_{n}$ are terms and $X$ is an $n$-ary second order variable). The connectives will be $\neg, \vee, \exists$, and $\exists^{2}$. Other connectives can be defined in the usual way.

The rules of this system will be:

1. Propositional Rules

(a) $\Gamma, A, \neg A$ for any atomic $A$

(b) From $\Gamma, \neg \phi$ and $\Gamma, \neg \psi$ conclude $\Gamma, \neg(\phi \vee \psi)$

(c) From $\Gamma, \phi$ conclude $\Gamma, \phi \vee \psi$ and $\Gamma, \psi \vee \phi$

(d) From $\Gamma, \phi$ and $\Gamma, \neg \phi$ conclude $\Gamma$

2. Quantifier rules

(a) From $\Gamma, \neg \phi(y)$ conclude $\Gamma, \neg \exists x \phi(x)$ if $y$ does not occur free in any formula of $\Gamma$

(b) From $\Gamma, \neg \phi(Y)$ conclude $\Gamma, \neg \exists^{2} X \phi(X)$ if $Y$ does not occur free in any formula of $\Gamma$

(c) From $\Gamma, \phi(t)$ conclude $\Gamma, \exists x \phi(x)$

(d) From $\Gamma, \phi(\lambda \vec{y} \cdot B)$ conclude $\Gamma, \exists^{2} X \phi(X)$

3. Equality rules (quantifier free)

- $\Gamma, t=t$ for any term $t$

- From $\Gamma, t_{1}=t_{2}$ conclude $\Gamma, t_{2}=t_{1}$ for any terms $t_{1}$ and $t_{2}$

- From $\Gamma, t_{1}=t_{2}$ and $\Gamma, \phi\left(t_{1}\right)$ conclude $\Gamma, \phi\left(t_{2}\right)$ for any terms $t_{1}$ and $t_{2}$

4. Arithmetical rules

(a) Quantifier-free defining equations for all primitive recursive relations and functions 
(b) From $\Gamma, \neg \phi(0)$ and $\Gamma, \phi(y), \neg \phi(S y)$ conclude $\Gamma, \neg \exists x \phi(x)$ if $y$ does not occur free in $\Gamma$

All other normal rules of second order arithmetic can be derived from these, for example:

$\frac{\Gamma, \phi \quad \Gamma, \neg \phi, \neg \neg \phi}{\Gamma, \neg \neg \phi}$

If $\Gamma=\left\{\phi_{1}, \ldots, \phi_{k}\right\}$ then $\neg \Gamma=\left\{\neg \phi_{1}, \ldots, \neg \phi_{k}\right\}$.

Intuitionistic logic and $H A^{2}$ will be given by a system of natural deduction with connectives $\forall, \exists, \exists^{2}, \vee$, and $\rightarrow(\exists$ and $\vee$ are redundant, but it is more convenient to include them; $\forall^{2}$ and $\wedge$ will not be needed, so they are excluded).

\section{Friedman-Dragalin Translation}

As noted above, a formula $\phi$ of $P A^{2}$ can be associated with a formula $\left.\left.\phi\right\urcorner\right\urcorner$ of $H A^{2}$ such that $\left.\left.P A^{2} \vdash \phi \Leftrightarrow H A^{2} \vdash \phi\right\urcorner\right\urcorner$. The embedding $E$ used here is simpler, although the result proved will be correspondingly weaker:

- $\phi^{E} \equiv \phi$ for atomic $\phi$

- $(\neg \phi)^{E} \equiv \phi^{E} \rightarrow \perp$

- $(\phi \vee \psi)^{E} \equiv \phi^{E} \vee \psi^{E}$

- $(\exists x \phi(x))^{E} \equiv \exists x \phi(x)^{E}$

- $(\exists X \phi(X))^{E} \equiv \exists X \phi(X)^{E}$

Given a fixed formula $\alpha$ of $H A^{2}$, a translation $F D(\alpha)$ of formulas within $H A^{2}$ can be defined so that $\alpha \rightarrow \phi^{F D(\alpha)}$ for every $\phi$ :

- $\phi^{F D(\alpha)} \equiv \phi\left(\right.$ for $\left.\phi=X t_{1} \ldots t_{n}\right)$

- $\phi^{F D(\alpha)} \equiv \phi \vee \alpha$ (for other atomic $\phi$ )

- $\perp^{F D(\alpha)} \equiv \alpha$

- $(\phi \rightarrow \psi)^{F D(\alpha)} \equiv \phi^{F D(\alpha)} \rightarrow \psi^{F D(\alpha)}$

- $(\phi \vee \psi)^{F D(\alpha)} \equiv \phi^{F D(\alpha)} \vee \psi^{F D(\alpha)}$

- $(\exists x \phi(x))^{F D(\alpha)} \equiv \exists x \phi(x)^{F D(\alpha)}$

- $(\exists X \phi(X))^{F D(\alpha)} \equiv \exists X \phi(X)^{F D(\alpha)}$

Note that $\left(X t_{1} \ldots t_{n}\right)^{F D(\alpha)}=X t_{1} \ldots t_{n}$ is not itself implied by $\alpha$ unless the range of $X$ is restricted to the range of $F D(\alpha)$. This is necessary to ensure that $F D(\alpha)$ commutes with substitution.

When composed these operations give a transformation $N$ from formulas of $P A^{2}$ to formulas of $H A^{2}$ : 
- $\phi^{N} \equiv \phi\left(\right.$ for $\left.\phi=X t_{1} \ldots t_{n}\right)$

- $\phi^{N} \equiv \phi \vee \alpha$ (for other atomic $\phi$ )

- $(\neg \phi)^{N} \equiv \phi^{N} \rightarrow \alpha$

- $(\phi \vee \psi)^{N} \equiv \phi^{N} \vee \psi^{N}$

- $(\exists x \phi(x))^{N} \equiv \exists x \phi(x)^{N}$

- $(\exists X \phi(X))^{N} \equiv \exists X \phi(X)^{N}$

Lemma 1. The $N$-translation commutes with substitution:

$$
\phi(\lambda \vec{y} \cdot B)^{N}=\left(\lambda Y . \phi(Y)^{N}\right)\left(\lambda \vec{y} \cdot B^{N}\right)
$$

or, equivalently:

$$
(\phi[\lambda \vec{y} \cdot B / Y])^{N}=\phi^{N}\left[\lambda \vec{y} \cdot B^{N} / Y\right]
$$

Proof. By induction on $\phi(Y)$. When $\phi(Y) \neq Y t_{1} \ldots t_{n}$, just apply the inductive hypothesis. When $\phi(Y)=Y t_{1} \ldots t_{n}$ then $\phi(\lambda \vec{y} . B)^{N}=\left(B t_{1} \ldots t_{n}\right)^{N}=B^{N} t_{1} \ldots t_{n}$ while $\left(\lambda Y . \phi(Y)^{N}\right)\left(\lambda y \cdot B^{N}\right)=\left(\lambda Y . Y t_{1} \ldots t_{n}\right)\left(\lambda \vec{y} \cdot B^{N}\right)=B^{N} t_{1} \ldots t_{n}$.

Lemma 2. If $d: \Gamma$ is a proof in $P A^{2}$ then $(\neg \Gamma)^{N} \vdash \alpha$ is provable in $H A^{2}$.

Proof. Proved by induction on the last step of $d$. The following two deductions will be used repeatedly:

$$
\begin{gathered}
\frac{\frac{\Gamma, \phi \Rightarrow \alpha}{\Gamma \Rightarrow \phi \rightarrow \alpha} \quad(\phi \rightarrow \alpha) \rightarrow \alpha \Rightarrow(\phi \rightarrow \alpha) \rightarrow \alpha}{} \\
\Gamma,(\phi \rightarrow \alpha) \rightarrow \alpha \Rightarrow \alpha \\
\frac{\Gamma \Rightarrow \phi \quad \phi \rightarrow \alpha \Rightarrow \phi \rightarrow \alpha}{\Gamma \Rightarrow(\phi \rightarrow \alpha) \rightarrow \alpha \Rightarrow \alpha} \\
\frac{\phi, \phi \rightarrow \alpha) \rightarrow \alpha) \rightarrow \alpha}{\Gamma, \phi \Rightarrow \alpha}
\end{gathered}
$$

- If $d$ is just the axiom $\Gamma, A, \neg A$ then either $(\neg A)^{N}=A \vee \alpha \rightarrow \alpha$ and $(\neg \neg A)^{N}=$ $(A \vee \alpha \rightarrow \alpha) \rightarrow \alpha$ or $(\neg A)^{N}=A \rightarrow \alpha$ and $(\neg \neg A)^{N}=(A \rightarrow \alpha) \rightarrow \alpha$. In either case, $\alpha$ follows by $\rightarrow E$.

- If $d$ concludes $\Gamma, \neg(\phi \vee \psi)$ from $\Gamma, \neg \phi$ and $\Gamma, \neg \psi$ then:

$$
\frac{(\neg \Gamma)^{N},\left(\phi^{N} \rightarrow \alpha\right) \rightarrow \alpha \Rightarrow \alpha}{\frac{(\neg \Gamma)^{N}, \phi^{N} \Rightarrow \alpha}{\frac{(\neg \Gamma)^{N}, \phi^{N} \vee \psi^{N} \Rightarrow \alpha}{(\neg \Gamma)^{N},\left(\phi^{N} \vee \psi^{N} \rightarrow \alpha\right) \rightarrow \alpha \Rightarrow \alpha}}}
$$

- If $d$ concludes $\Gamma, \phi \vee \psi$ from $\Gamma, \phi$ (the case for $\Gamma, \psi$ is similar) then: 


$$
\frac{\left(\phi^{N} \vee \psi^{N}\right) \rightarrow \alpha \Rightarrow\left(\phi^{N} \vee \psi^{N}\right) \rightarrow \alpha \quad \frac{\phi^{N} \Rightarrow \phi^{N}}{\phi^{N} \Rightarrow \phi^{N} \vee \psi^{N}}}{\frac{\left(\phi^{N} \vee \psi^{N}\right) \rightarrow \alpha, \phi^{N} \Rightarrow \alpha}{\left(\phi^{N} \vee \psi^{N}\right) \rightarrow \alpha \Rightarrow \phi^{N} \rightarrow \alpha}}
$$

and

$$
\frac{\frac{(\neg \Gamma)^{N}, \phi^{N} \rightarrow \alpha \Rightarrow \alpha}{(\neg \Gamma)^{N} \Rightarrow\left(\phi^{N} \rightarrow \alpha\right) \rightarrow \alpha} \quad\left(\phi^{N} \vee \psi^{N}\right) \rightarrow \alpha \Rightarrow \phi^{N} \rightarrow \alpha}{(\neg \Gamma)^{N},\left(\phi^{N} \vee \psi^{N}\right) \rightarrow \alpha \Rightarrow \alpha}
$$

- If $d$ concludes $\Gamma$ from $\Gamma, \phi$ and $\Gamma, \neg \phi$ then:

$$
\frac{\frac{(\neg \Gamma)^{N}, \phi^{N} \rightarrow \alpha \Rightarrow \alpha}{(\neg \Gamma)^{N} \Rightarrow\left(\phi^{N} \rightarrow \alpha\right) \rightarrow \alpha} \quad \frac{(\neg \Gamma)^{N},\left(\phi^{N} \rightarrow \alpha\right) \rightarrow \alpha \Rightarrow \alpha}{(\neg \Gamma)^{N} \Rightarrow\left(\left(\phi^{N} \rightarrow \alpha\right) \rightarrow \alpha\right) \rightarrow \alpha}}{(\neg \Gamma)^{N} \Rightarrow \alpha}
$$

- If $d$ concludes $\Gamma, \neg \exists x \phi(x)$ from $\Gamma, \neg \phi(y)$ then:

$$
\frac{(\neg \Gamma)^{N},\left(\phi(y)^{N} \rightarrow \alpha\right) \rightarrow \alpha \Rightarrow \alpha}{\frac{(\neg \Gamma)^{N}, \phi(y)^{N} \Rightarrow \alpha}{(\neg \Gamma)^{N}, \exists x \phi(x)^{N} \Rightarrow \alpha} \quad \exists x \phi(x)^{N} \Rightarrow \exists x \phi(x)^{N}}
$$

- If $d$ concludes $\Gamma, \exists x \phi(x)$ from $\Gamma, \phi(t)$ then:

$$
\frac{\frac{\phi(t)^{N} \Rightarrow \phi(t)^{N}}{\phi(t)^{N} \Rightarrow \exists x \phi(x)^{N}} \quad \exists x \phi(x)^{N} \rightarrow \alpha \Rightarrow \exists x \phi(x)^{N} \rightarrow \alpha}{\frac{\exists x \phi(x)^{N} \rightarrow \alpha, \phi(t)^{N} \Rightarrow \alpha}{\exists x \phi(x)^{N} \rightarrow \alpha \Rightarrow \phi(t)^{N} \rightarrow \alpha}}
$$

and

$$
\frac{\frac{(\neg \Gamma)^{N}, \phi(t)^{N} \rightarrow \alpha \Rightarrow \alpha}{(\neg \Gamma)^{N} \Rightarrow\left(\phi(t)^{N} \rightarrow \alpha\right) \rightarrow \alpha} \quad \exists x \phi(x)^{N} \rightarrow \alpha \Rightarrow \phi(t)^{N} \rightarrow \alpha}{(\neg \Gamma)^{N}, \exists x \phi(x)^{N} \rightarrow \alpha \Rightarrow \alpha}
$$

- If $d$ concludes $\Gamma, \neg \exists x \phi(x)$ from $\Gamma, \phi(0)$ and $\Gamma, \neg \phi(y), \phi(S y)$ then:

$$
\begin{aligned}
& \frac{(\neg \Gamma)^{N}, \phi(y)^{N} \rightarrow \alpha,\left(\phi(S y)^{N} \rightarrow \alpha\right) \rightarrow \alpha \Rightarrow \alpha}{\frac{(\neg \Gamma)^{N}, \phi(y)^{N} \rightarrow \alpha, \phi(S y)^{N} \Rightarrow \alpha}{(\neg \Gamma)^{N}, \phi(y)^{N} \rightarrow \alpha \Rightarrow \phi(S y)^{N} \rightarrow \alpha}} \\
& \frac{(\neg \Gamma)^{N},\left(\phi(0)^{N} \rightarrow \alpha\right) \rightarrow \alpha \Rightarrow \alpha}{\frac{(\neg \Gamma)^{N}, \phi(0)^{N} \Rightarrow \alpha}{(\neg \Gamma)^{N} \Rightarrow \phi(0)^{N} \rightarrow \alpha}(\neg \Gamma)^{N}, \phi(y)^{N} \rightarrow \alpha \Rightarrow \phi(S y)^{N} \rightarrow \alpha} \\
& \frac{(\neg \Gamma)^{N} \Rightarrow \forall x\left[\phi(x)^{N} \rightarrow \alpha\right]}{\frac{(\neg \Gamma)^{N} \Rightarrow \phi(y)^{N} \rightarrow \alpha}{(\neg \Gamma)^{N}, \phi(y)^{N} \Rightarrow \alpha} \Rightarrow \phi(y)^{N}}
\end{aligned}
$$


and

$$
\frac{(\neg \Gamma)^{N}, \phi(y)^{N} \Rightarrow \alpha \quad \exists x \phi(x)^{N} \Rightarrow \exists x \phi(x)^{N}}{\frac{(\neg \Gamma)^{N}, \exists x \phi(x)^{N} \Rightarrow \alpha}{(\neg \Gamma)^{N},\left(\exists x \phi(x)^{N} \rightarrow \alpha\right) \rightarrow \alpha \Rightarrow \alpha}}
$$

- Suppose $d: \phi$. Then $\phi$ is also an axiom of $H A^{2}$, so:

$$
\frac{\phi \rightarrow \alpha \Rightarrow \phi \rightarrow \alpha \quad \Rightarrow \phi}{\phi \rightarrow \alpha \Rightarrow \alpha}
$$

- If $d$ concludes $\Gamma, \exists X \phi(X)$ from $\Gamma, \phi(\lambda \vec{y} \cdot B)$ then:

$$
\frac{\exists X \phi(X)^{N} \rightarrow \alpha \Rightarrow \exists X \phi(X)^{N} \rightarrow \alpha \quad \frac{\phi(\lambda \vec{y} \cdot B)^{N} \Rightarrow \phi(\lambda \vec{y} \cdot B)^{N}}{\phi(\lambda \vec{y} \cdot B)^{N} \Rightarrow \exists X \phi(X)^{N}}}{\frac{\exists X \phi(X)^{N} \rightarrow \alpha, \phi(\lambda \vec{y} \cdot B)^{N} \Rightarrow \alpha}{\exists X \phi(X)^{N} \rightarrow \alpha \Rightarrow \phi(\lambda \vec{y} \cdot B)^{N} \rightarrow \alpha}}
$$

and

$$
\frac{\frac{(\neg \Gamma)^{N}, \phi(\lambda \vec{y} \cdot B)^{N} \rightarrow \alpha \Rightarrow \alpha}{\exists X \phi(X)^{N} \rightarrow \alpha \Rightarrow \phi(\lambda \vec{y} \cdot B)^{N} \rightarrow \alpha} \quad(\neg \Gamma)^{N} \Rightarrow\left(\phi(\lambda \vec{y} \cdot B)^{N} \rightarrow \alpha\right) \rightarrow \alpha}{(\neg \Gamma)^{N}, \exists X \phi(X)^{N} \rightarrow \alpha \Rightarrow \alpha}
$$

- If $d$ concludes $\Gamma, \neg \exists X \phi(X)$ from $\Gamma, \neg \phi(Y)$ then:

$$
\frac{(\neg \Gamma)^{N},\left(\phi(Y)^{N} \rightarrow \alpha\right) \rightarrow \alpha \Rightarrow \alpha}{\left.\frac{(\neg \Gamma)^{N}, \phi(Y)^{N} \Rightarrow \alpha}{(\neg X)^{N}, \exists X \phi(X)^{N} \Rightarrow \alpha}\right)^{N} \Rightarrow \exists X \phi(X)^{N}}
$$

\section{$4 \mathrm{HRO}^{2}$}

The language of $H R O^{2}$ is arithmetic augmented by definitions equating every hereditarily partially recursive function of finite type with a number. More precisely, each partially recursive function is associated with its Gödel number $x$, and $\{x\}(y)$ is used to denote the (possibly undefined) value of the function associated with $x$ when applied to $y$; when $\{x\}(y)$ is defined, this is denoted $\{x\}(y) \downarrow$. For technical reasons, 0 should be the constantly 0 function.

The functionals in question are the second order functionals of system F; the set $T$ of types of these functionals is given by:

- The type 0 of the natural numbers is in $T$

- If $\sigma, \tau \in T$ then $\sigma \rightarrow \tau \in T$

- For any $n$, a variable type $\alpha_{n} \in T$ 
- If $\sigma, \tau \in T$ then $\sigma \times \tau \in T$

- If $\sigma\left[\alpha_{n}\right] \in T$ then $\forall \alpha_{n} . \sigma\left[\alpha_{n}\right] \in T$

- If $\sigma\left[\alpha_{n}\right] \in T$ then $\exists \alpha_{n} . \sigma\left[\alpha_{n}\right] \in T$

$H R O^{2}$ is given by associating to each $\sigma \in T$ a set of numbers $V_{\sigma}$ (representing the numbers denoting functions of that type) and to each type variable $\alpha$ a variable $V_{\alpha}$ ranging over the sets $V_{\sigma}$ :

- All numbers are in $V_{0}$

- If $\alpha_{n} \in T$ is a type variable then there is a corresponding set variable $V_{\alpha_{n}}$

- $x \in V_{\sigma \rightarrow \tau}$ if for any $y \in V_{\sigma},\{x\}(y) \in V_{\tau}$

- $x \in V_{\sigma \times \tau}$ if $(x)_{0} \in V_{\sigma}$ and $(x)_{1} \in V_{\tau}$

- $x \in \forall \alpha_{n} . \sigma\left[\alpha_{n}\right]$ if for any $V \in T, x \in V_{\sigma\left[\alpha_{n}\right]}\left[V / V_{\alpha_{n}}\right]$

- $x \in \exists \alpha_{n} . \sigma\left[\alpha_{n}\right]$ if there is some $V \in T$ such that $x \in V_{\sigma\left[\alpha_{n}\right]}\left[V / V_{\alpha_{n}}\right]$

Full details of the construction are given in [Troelstra, 1973].

\section{Realizability}

The modified realizability $H R O^{2}$-mr assigns a predicate, Realizes ${ }_{\phi}$ from $H R O^{2}$, to each formula $\phi$ of $H A^{2}$. A number realizes a formula $\phi$ when the term it represents executes a computation which demonstrates the truth of the formula. It is then possible to assign a specific term to a deduction $d$ which realizes the conclusion of $d$.

In order to define the realizability, it is first necessary to define a predicate which is satisfied when a number encodes a functional of the appropriate type to realize a formula. Following the notation in [Troelstra, 1973], a unary second order variable $U_{X}^{1}$ of $H R O^{2}$ is uniquely associated to each second order variable $X$ of $H A^{2}$. For technical reasons, the set denoted by $U_{X}^{1}$ must contain 0 , so $\exists U_{X}^{1}$ will represent quantification only over those formulae which are satisfied by 0 . Then:

1. Type $\operatorname{set}(x) \equiv[x=x]$ where $x$ is not free in $s$ or $t$

2. $\operatorname{Type}_{X \vec{t}}(x) \equiv U_{X}^{1} x$

3. $\operatorname{Type}_{\phi \vee \psi}(x) \equiv\left((x)_{0}=0 \rightarrow \operatorname{Type}_{\phi}\left((x)_{1}\right)\right) \wedge\left((x)_{0} \neq 1 \rightarrow \operatorname{Type}_{\psi}\left((x)_{1}\right)\right)$

4. $\operatorname{Type}_{\phi \rightarrow \psi}(x) \equiv \forall y\left(\operatorname{Type}_{\phi}(y) \rightarrow\{x\}(y) \downarrow \wedge \operatorname{Type}_{\psi}(\{x\}(y))\right)$

5. $\operatorname{Type}_{\exists y \phi(y)}(x) \equiv \operatorname{Type}_{\phi\left((x)_{0}\right)}\left((x)_{1}\right)$

6. $\operatorname{Type}_{\exists X^{n} \phi(X)}(x) \equiv \exists U_{X}^{1} \operatorname{Type}_{\phi(X)}(x)$

An $n+1$-ary second order variable of $H R O^{2}, X^{*}$, must be uniquely associated to each $n$-ary second order variable $X$ of $H A^{2}$. Then the realizability is given by: 
1. Realizes $s_{s=t}(x) \equiv[s=t]$

2. Realizes ${ }_{X \vec{t}}(x) \equiv X^{*}(x, \vec{t}) \wedge \operatorname{Type}_{X \vec{t}}(x)$

3. Realizes $_{\phi \vee \psi}(x) \equiv\left((x)_{0}=0 \rightarrow \operatorname{Realizes}_{\phi}\left((x)_{1}\right)\right)$ $\wedge\left((x)_{0} \neq 0 \rightarrow\right.$ Realizes $\left._{\psi}\left((x)_{1}\right)\right)$

4. Realizes $_{\phi \rightarrow \psi}(x) \equiv \operatorname{Type}_{\phi \rightarrow \psi}(x)$ $\wedge \forall y\left(\operatorname{Realizes}_{\phi}(y) \rightarrow\{x\}(y) \downarrow \wedge \operatorname{Realizes}_{\psi}(\{x\}(y))\right)$

5. Realizes $\exists_{\exists \phi(y)}(x) \equiv$ Realizes $_{\phi\left((x)_{0}\right)}\left((x)_{1}\right)$

6. Realizes $\exists X^{n} \phi(X)(x) \equiv \exists Y^{*} \exists U_{Y}^{1} \operatorname{Realizes}_{\phi(Y)}(x)$

The rules of $P A^{2}$ are not sound for this realizability, but their $N$-translations are; for instance, there is no term corresponding to the axiom $\phi \vee \neg \phi$, but $\phi^{N} \rightarrow \alpha, \phi^{N} \rightarrow$ $\alpha \rightarrow \alpha \vdash \alpha$ does correspond to a term. In particular, if $\alpha=\exists x A(x)$ where $A$ is a primitive recursive relation then we say $x P A^{2}$-realizes a formula $\phi$ of $P A^{2}$ if Realizes $_{\phi^{N}}(x)$. Note that Realizes $\alpha(x) \equiv A\left((x)_{0}\right)$, so

$$
\operatorname{Type}_{(\neg \phi)^{N}}(x) \equiv \forall y\left(\operatorname{Type}_{\phi^{N}}(y) \rightarrow\{x\}(y) \downarrow\right)
$$

$\operatorname{Realizes}_{(\neg \phi)^{N}} \equiv \operatorname{Type}_{(\neg \phi)^{N}}(y) \wedge \forall y\left(\operatorname{Realizes}_{\phi^{N}}(y) \rightarrow\{x\}(y) \downarrow \wedge A\left((\{x\}(y))_{0}\right)\right)$

$\alpha$ may have additional free variables so long as they are renamed to be different from the eigenvalues in any application of the induction or $\forall$ rules. Any free variables other than $x$ will in general also be a free variable in Realizes $\phi$. In this case, Realizes $_{\phi}(t)$ means that $t$ is a term (possibly with the same free variables as $A$ ) realizing $\phi$ for every value of those variables.

In general, we use $\alpha_{\phi}$ for a first order variable intended to satisfy $\operatorname{Type}_{\phi^{N}}\left(\alpha_{\phi}\right)$ and when $\Gamma=\left\{\phi_{1}, \ldots, \phi_{k}\right\}$ is a sequent, we intend $\alpha_{\Gamma}=\left(\alpha_{\phi_{1}}, \ldots, \alpha_{\phi_{k}}\right)$ to be a sequence of variables such that Type ${ }_{\phi_{i}^{N}}\left(\alpha_{\phi_{i}}\right)$.

Lemma 3. 1. Write $[*]$ for $\left[\lambda x \operatorname{Type}_{B \vec{y}}(x) / U_{X}^{1}\right]$. Then

$$
\operatorname{Type}_{\phi(X \vec{t})}[*]=\text { Type }_{\phi(B \vec{t})}
$$

2. Write [†] for $\left[\lambda x \lambda \vec{y} \operatorname{Realizes}_{B \vec{y}}(x) / X^{*}\right]$. Then

$$
\text { Realizes }_{\phi(X \vec{t})}(x)[*][\dagger] \leftrightarrow \text { Realizes }_{\phi(B \vec{t})}(x)
$$

Proof. 1. Proved by a straightforward induction on $\phi(X)$. When $\phi(X)=X \vec{t}$ then

$$
\operatorname{Type}_{X \vec{t}}(x)[*]=U_{X}^{1}(x)[*]=\operatorname{Type}_{B \vec{t}}(x)
$$

The other cases just apply the inductive hypothesis. 
2. Proved by induction on $\phi(X)$. When $\phi(X)=X \vec{t}$ then

$$
\begin{aligned}
\text { Realizes }_{X \vec{t}}(x)[*][\dagger] & =X^{*}(x, \vec{t})[\dagger] \wedge \operatorname{Type}_{X \vec{t}}(x)[*] \\
& =\operatorname{Realizes}_{B \vec{t}}(x) \wedge \operatorname{Type}_{B \vec{t}}(x) \\
& \leftrightarrow \operatorname{Realizes}_{B \vec{t}}(x)
\end{aligned}
$$

The other cases just apply the inductive hypothesis.

A deduction of $\Gamma \vdash \phi$ in $H A^{2}$ can be assigned a term of $H R O^{2}$-mr with free variables corresponding to the elements of $\Gamma$ and which realizes $\phi$ whenever the free variables realize the corresponding elements of $\Gamma$. If $\Gamma$ or $\phi$ has free variables, those will in general also be free variables of the term, and for any assignment of values to those variables, the term will realize $\phi$. For axioms, the term is 0 , and, for example, the deduction $\frac{d: \Gamma, \phi \Rightarrow \psi}{\Gamma \Rightarrow \phi \rightarrow \psi}$ becomes $\lambda$ a.t where $t$ is the term correspond to $d$.

Free variables which appear in the premise but not conclusion of a proof rule can be eliminated in the corresponding terms. Specifically, if $d$ applies $\forall I, \forall^{2} I, \exists I, \vee E$, $\rightarrow E$, or $\exists E$ to $d_{0}$ (and $d_{1}$ and $d_{2}$ when appropriate) and $x$ or $X^{n}$ is a free variable appearing in $d_{0}, d_{1}$, or $d_{2}$ but not in $d$ then if $t_{0}\left(t_{1}, t_{2}\right)$ are the corresponding terms, replace all occurrences of $x$ with 0 and all occurrences of $X^{n}$ with $\lambda \vec{y} .\left(\forall X^{0}\right) X$ before constructing $t$. For instance suppose $d_{0}: \Gamma \Rightarrow \psi \rightarrow \phi(x, X)$ and $d_{1}: \Sigma \Rightarrow \psi$ with $x$ and $X^{n}$ variable not appearing in $\psi, \Gamma$, or $\Sigma$. Then the corresponding term is $t_{0}[0 / x]\left[\lambda \vec{y} \cdot\left(\forall X^{0}\right) X / X\right]\left(t_{1}\right)$.

Theorem 1. If $d$ is a deduction of $\Gamma$ in $P A^{2}$ then there is a term $F_{d}$ with free variables among $\alpha_{\neg \Gamma} \cup \mathrm{FV}(\Gamma) \cup \mathrm{FV}(\alpha)$ such that if $\operatorname{Type}_{(\neg \phi)^{N}}\left(\alpha_{\neg \phi}\right)$ for each $\phi \in \Gamma$ then $H A^{2}$ proves $\left(\lambda \alpha_{\neg \Gamma} \cdot F_{d}\right)\left(\alpha_{\neg \Gamma}\right) \downarrow$ and if Realizes ${ }_{(\neg \phi)^{N}}\left(\alpha_{\neg \phi}\right)$ holds for each $\phi \in \Gamma$ then $H A^{2}$ proves $A\left(\left(\left(\lambda \alpha_{\neg \Gamma} \cdot F_{d}\right)\left(\alpha_{\neg \Gamma}\right)\right)_{0}\right)$.

Proof. Since $d$ is a deduction of $\Gamma$ in $P A^{2}$, there is a deduction $D$ of $(\neg \Gamma)^{N} \vdash \exists x A(x)$ in $H A^{2}$. The theorem could be proved by simply appealing to the realization given in [Troelstra, 1973]. However this can also be proved directly by defining the term inductively on the last step of $d$; the appropriate can be easily found by taking the $H A^{2}$ deduction corresponding to an inference in $P A^{2}$ and applying the Curry-Howard isomorphism.

It will be necessary to remove extraneous free variables during this process. If $d$ applies the cut rule or the first or second order $\exists$ rules, there may be free first or second order variables which appear in the premises but not the conclusion. If $d: \phi$ is an application of one of these three rules to $d_{1}: \phi_{1}$ (and $d_{2}: \phi_{2}$ in the case of cut) and $x$ or $X$ is a free variable in $\phi_{1}$ (and $\phi_{2}$ in the case of cut) which does not appear in $\phi$ then the inference

$$
\begin{aligned}
& d_{1}[0 / x]\left[\lambda \vec{y} \cdot\left(\forall X^{0}\right) X\right] \quad\left(d_{2}[0 / x]\left[\lambda \vec{y} \cdot\left(\forall X^{0}\right) X\right]\right) \\
& \hline d
\end{aligned}
$$

is also a valid inference. The corresponding terms, $t_{1}[0 / x]\left[\lambda \vec{y} .\left(\forall X^{0}\right) X\right]$ and $t_{2}[0 / x]\left[\lambda \vec{y} \cdot\left(\forall X^{0}\right) X\right]$ should be used in the inductive construction of $t$. 
- $d$ is any of the quantifier free axioms. Then $\Gamma=\left\{\phi_{1}, \ldots, \phi_{k}\right\}$ and at least one $\phi_{i}$ must be true, therefore it is never possible for $\alpha_{\neg \Gamma}$ to realize $\neg \Gamma$, so

$$
F_{d} \equiv 0
$$

- $d$ is an axiom of the form $\Gamma, A, \neg A$. Then:

$$
F_{d} \equiv\left\{\alpha_{\neg \neg A}\right\}\left(\alpha_{\neg A}\right)
$$

- $d$ concludes $\Gamma, \phi \vee \psi$ from $d^{\prime}: \Gamma, \phi$ (the case for $d^{\prime}: \Gamma, \psi$ is similar). Then:

$$
F_{d} \equiv\left(\lambda \alpha_{\neg \phi} \cdot F_{d^{\prime}}\right)\left(\left\ulcorner\lambda \alpha_{\phi} \cdot\left\{\alpha_{\neg(\phi \vee \psi)}\right\}\left(\left\langle 0, \alpha_{\phi}\right)\right\urcorner\right)\right.
$$

- $d$ concludes $\Gamma, \neg(\phi \vee \psi)$ from $d_{0}: \Gamma, \neg \phi$ and $d_{1}: \Gamma, \neg \psi$. Then primitive recursion can be used to define by cases:

$$
F^{\prime} \equiv\left\ulcorner\left\{\begin{array}{ll}
\left(\lambda \alpha_{\neg \neg \phi} \cdot F_{0}\right)\left(\left\ulcorner\lambda \alpha_{\neg \phi} \cdot\left\{\alpha_{\neg \phi}\right\}\left(\left(\alpha_{\phi \vee \psi}\right)_{1}\right)\right\urcorner\right) & \text { if }\left(\alpha_{\phi \vee \psi}\right)_{0}=0 \\
\left(\lambda \alpha_{\neg \neg \psi} \cdot F_{1}\right)\left(\left\ulcorner\lambda \alpha_{\neg \psi} \cdot\left\{\alpha_{\neg \psi}\right\}\left(\left(\alpha_{\phi \vee \psi}\right)_{1}\right)\right\urcorner\right) & \text { if }\left(\alpha_{\phi \vee \psi}\right)_{0} \neq 0
\end{array}\right\urcorner\right.
$$

and define

$$
F_{d} \equiv\left\{\alpha_{\neg \neg(\phi \vee \psi)}\right\}\left(\left\ulcorner\lambda \alpha_{\phi \vee \psi} . F^{\prime}\right\urcorner\right)
$$

- $d$ concludes $\Gamma, \exists x \phi(x)$ from $d^{\prime}: \Gamma, \phi(t)$. If $t$ has any free variables that do not occur in the conclusion the should be replaced with 0 in $F_{d^{\prime}}$. Then:

$$
F_{d}=\left(\lambda \alpha_{\neg \phi(t)} \cdot F_{d^{\prime}}\right)\left(\left\ulcorner\lambda \alpha_{\phi(t)} \cdot\left\{\alpha_{\neg \exists x \phi(x)}\right\}\left(\left\langle t, \alpha_{\phi(t)}\right\rangle\right)\right\urcorner\right)
$$

- $d$ concludes $\Gamma, \neg \exists x \phi(x)$ from $d^{\prime}: \Gamma, \neg \phi(y)$. Then $F_{d^{\prime}}$ is a term which may contain $y$ free and $y$ does not occur free in $\Gamma$. So:

$$
\begin{aligned}
F_{d} \equiv & \left\{\alpha_{\neg \neg \exists x \phi(x)}\right\}\left(\left\ulcorner\lambda \alpha_{\exists x \phi(x)} \cdot\left(\lambda y \lambda \alpha_{\neg \neg \phi(y)} \cdot F_{d^{\prime}}\right)\right.\right. \\
& \left.\left.\left(\left(\alpha_{\exists x \phi(x)}\right)_{0}\right)\left(\lambda \alpha_{\neg \phi(y)} \cdot\left\{\alpha_{\neg \phi(y)}\right\}\left(\left(\alpha_{\exists x \phi(x)}\right)_{1}\right)\right)\right\urcorner\right)
\end{aligned}
$$

- $d$ derives $\Gamma$ from $d_{0}: \Gamma, \neg \phi$ and $d_{1}: \Gamma, \phi$. Replace any free variables which appear in $d_{0}$ and $d_{1}$ but not in $d$ with 0 (for first order variables) and $\left(\forall X^{0}\right) X$ (for second order variables). Then:

$$
F_{d} \equiv\left(\lambda \alpha_{\neg \neg \phi} . F_{0}\right)\left(\left\ulcorner\lambda \alpha_{\neg \phi} . F_{1}\right\urcorner\right)
$$

- $d$ is a deduction of $\Gamma, \neg \exists x \phi(x)$ from $d_{0}: \Gamma, \neg \phi(0)$ and $d_{1}: \Gamma, \phi(y), \neg \phi(S y)$. Then construct a function $h$ by primitive recursion:

$$
\begin{aligned}
h(0) \equiv\left\ulcorner\lambda \alpha_{\phi(0)} \cdot\left(\lambda \alpha_{\neg \neg \phi(0)} \cdot F_{d_{0}}\right)\left(\lambda \alpha_{\neg \phi(0)} \cdot\left\{\alpha_{\neg \phi(0)}\right\}\left(\alpha_{\phi(0)}\right)\right)\right\urcorner \\
h(S y) \equiv\left\ulcorner\left(\lambda \alpha_{\neg \phi(y)} \cdot \lambda \alpha_{\phi(S y)} \cdot\left(\lambda \alpha_{\neg \neg \phi(S y)} \cdot F_{d_{1}}\right)\right.\right. \\
\left.\left(\lambda \alpha_{\neg \phi(S y)} \cdot\left\{\alpha_{\neg \phi(S y)}\right\}\left(\alpha_{\phi(S y)}\right)\right)(h(y))\right\urcorner
\end{aligned}
$$

Note that Realizes $(\neg \phi(n))^{N}(h(n))$ for every $n$.

Then:

$$
F_{d} \equiv\left\{\alpha_{\neg \neg \exists x \phi(x)}\right\}\left(\lambda \alpha_{\exists x \phi(x)} \cdot\left\{h\left(\left(\alpha_{\exists x \phi(x)}\right)_{0}\right)\right\}\left(\left(\alpha_{\exists x \phi(x)}\right)_{1}\right)\right)
$$


- $d$ is a deduction of $\Gamma, \exists X \phi(X)$ from $d^{\prime}: \Gamma, \phi(\lambda \vec{y} . B)$

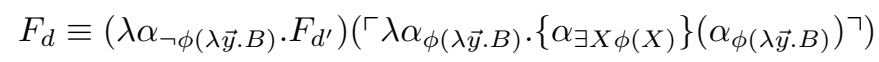

Free variables appearing in $d^{\prime}$ but not $d$ should be replaced.

- $d$ is a deduction of $\Gamma, \neg \exists X \phi(X)$ from $d^{\prime}: \Gamma, \neg \phi(Y)$ then:

$$
\begin{aligned}
F_{d} \equiv & \left\{\alpha_{\neg \neg \exists X \phi(X)}\right\}\left(\left\ulcorner\lambda \alpha _ { \exists X \phi ( X ) } \cdot \left[\left(\lambda \alpha_{\phi(Y)} \cdot\left(\lambda \alpha_{\neg \neg \phi(Y)} \cdot F_{d^{\prime}}\right)\right.\right.\right.\right. \\
& \left.\left.\left.\left.\left(\left\ulcorner\lambda \alpha_{\neg \phi(Y)} \cdot\left\{\alpha_{\neg \phi(Y)}\right\}\left(\alpha_{\phi(Y)}\right)\right\urcorner\right)\right)\left(\alpha_{\exists X \phi(X)}\right)\right]\right\urcorner\right)
\end{aligned}
$$

Theorem 2. If $d$ is a deduction of $\exists x A(x)$ where $A(x)$ is primitive recursive then it is possible to construct a term tof $H R O^{2}$ with the same free variables as $\exists x A(x)$ such that $A(t)$ holds for every value of those variables.

Proof. Cut $d$ with a hypothesis $h: \neg \exists x A(x)$; this gives a proof $d^{\prime}$ of the empty sequent. Let $F_{h}=\left\{\alpha_{\neg \neg \exists x A(x)}\right\}\left(\left\ulcorner\lambda \alpha_{\exists x A(x)} \cdot \alpha_{\exists x A(x)}\right\urcorner\right)$. Then, applying the previous theorem, $t=F_{d^{\prime}}$ is a term with no free variables, and therefore $A\left((t)_{0}\right)$.

If $A$ has free variables other than $x$, they will also, in general, be free variables in the corresponding term, so as an easy corollary we have:

Theorem 3. If $f$ is some function and $A$ is primitive recursive relation symbol representing the graph of $f$ and $P A^{2} \vdash \forall y \exists x A(y, x)$ then there is a term $t$ in $H R O^{2}$ with free variable $y$ such that $f=\lambda$ y.t.

Proof. Since $P A^{2}$ proves $\forall y \exists x A(y, x)$, there is also a $P A^{2}$ deduction $d$ of $\exists x A(y, x)$. Then the term $\left(F_{d}\right)_{0}$ given by the previous theorem suffices.

\section{References}

[Avigad, 2000] Avigad, J. (2000). A realizability interpretation for classical arithmetic. In Buss, Hájek, and Pudlák, editors, Logic Colloquium '98, number 13 in Lecture Notes in Logic, pages 57-90. AK Peters.

[Dragalin, 1980] Dragalin, A. (1980). New forms of realizability and Markov's rule (Russian). Doklady, 251:534-537. Translation SM 21, pp. 461-464.

[Friedman, 1978] Friedman, H. (1978). Higher Set Theory, volume 669 of Springer Lecture Notes, chapter Classically and Intuitionistically Provably Recursive Functions, pages 21-27. Springer.

[Kreisel, 1959] Kreisel, G. (1959). Interpretation of analysis by means of functionals of finite type. In Heyting, A., editor, Constructivity in Mathematics, pages 101-128. North-Holland. 
[Kreisel, 1962] Kreisel, G. (1962). On weak completeness of intuitionistic predicate logic. Journal of Symbolic Logic, 27:139-158.

[Schwichtenberg, 1977] Schwichtenberg, H. (1977). The Handbook of Mathematical Logic, chapter Proof theory: Some aspects of cut-elimination, pages 867-895. North-Holland.

[Troelstra, 1973] Troelstra, A., editor (1973). Metamathematical Investigation of Intuitionistic Arithmetic and Analysis. Springer. With contributions by A.S. Troelstra, C.A. Smoryński, J.I. Zucker and W.A. Howard. 